\title{
Entropy Generation and Bejan Number Analysis of MHD Casson Fluid Flow in a Micro-Channel with Navier Slip and Convective Boundary Conditions
}

\author{
M. Venkateswarlu ${ }^{1 *}$, P. Bhaskar ${ }^{2}$

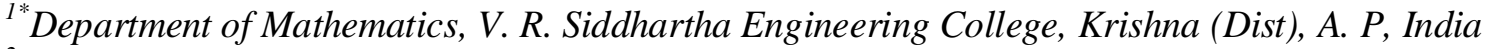 \\ ${ }^{2}$ Department of Mathematics, Shri Venkateshwara University, Gajraula, Amroha (Dist), U. P, India
}

Received: 5 April 2020; Received in revised form: 8 June 2020; Accepted: 22 June 2020; Published online 28 September 2020

(C) Published at www.ijtf.org

\begin{abstract}
The analysis of MHD flow has been a concern of consideration for research scientists and engineers. In this treatise, the steady MHD flow of an incompressible and electrically conducting Casson fluid in a micro-channel with heat generation and viscous dissipation, in the presence of hydrodynamic slip and convective boundary conditions, is examined. Exact solutions of non-dimensional steady governing equations are obtained in closed form. Transient fluid velocity, temperature, entropy generation, and Bejan number are depicted by the line graphs whereas rate of heat transfer and skin-friction coefficient are computed in tabular form for pertinent flow parameters. It is established that the entropy generation rate and Bejan number increases for increasing values of the Casson parameter and heat generation parameter. In particular, the Casson parameter accelerates the skin-friction coefficient while it provides resistance to the rate of heat transfer near the channel walls. Casson fluid finds significant applications in biomechanics, polymer processing industries, and food processing.
\end{abstract}

Keywords: Hydrodynamic Slip; Entropy generation; Bejan number; Micro-channel; Casson fluid.

\section{Nomenclature}

$\begin{array}{llll}a & \text { distance between two parallel plates } & E c & \text { Eckert number } \\ B e & \text { Bejan number } & H & \text { heat generation parameter } \\ B_{0} & \text { uniform magnetic field } & k & \text { permeability of the porous medium } \\ B_{1} & \text { Biot number for upper wall } & K & \text { permeability parameter } \\ B_{2} & \text { Biot number for lower wall } & M & \text { Hartmann number } \\ c_{p} & \text { specific heat at constant pressure } & N s & \text { entropy generation rate }\end{array}$

Corresponding e-mail: mvsr2010@gmail.com (M. Venkateswarlu) 
M. Venkateswarlu et al.

International Journal of Thermofluid Science and Technology (2020), Volume 7, Issue 4, Paper No. 070403

\begin{tabular}{llll}
\hline$P$ & pressure gradient & $v$ & fluid velocity in $y$-direction \\
$P e$ & Peclet number & $W$ & characteristic temperature ratio \\
$P r$ & Prandtl number & $\beta$ & Casson parameter \\
$Q_{0}$ & heat source coefficient & $\beta_{1}$ & upper wall slip parameter \\
$q_{w}$ & heat flux & $\beta_{2}$ & lower wall slip parameter \\
$R e$ & Reynolds number & $v$ & kinematic coefficient of viscosity \\
$T$ & fluid temperature & $\rho$ & fluid density \\
$T_{a}$ & ambient temperature & $\sigma$ & fluid electrical conductivity \\
$T_{h}$ & hot fluid temperature & $\tau$ & skin-friction coefficient \\
$u$ & fluid velocity in $x-$ direction & $\tau_{w}$ & shear stress \\
$U$ & non-dimensional velocity & $\eta$ & a scaled coordinate \\
$V_{0}$ & uniform suction/injection velocity & $\theta$ & a scaled temperature
\end{tabular}

\section{Introduction}

MHD (Hydromagnetic) is the study of the microscopic interaction of electrically conducting liquid and gas with the magnetic field. It has several significant applications in science and engineering, for instance, astrophysics, aerospace, solar energy collectors, nuclear reactors, wind energy, electromagnetics, transformers, geomechanics, oceanography, electrical heaters, plasma confinement, magnetic drug targeting, geophysics, etc. The groundwork of research of entropy generation goes back to Clausius and Kelvins investigations on the irreversibility attitudes of the second law of thermodynamics. On the other hand, the entropy generation resulting from temperature variations has remained untreated by classical thermodynamics. The second law analysis is significant because it is one of the techniques used for concluding the achievement of engineering processes. Since entropy generation is the measure of the extermination of accessible production of the system, the evaluation of the effective factors exciting the entropy generation is significant in promoting the system performances. Rapid development in science and technology has led to the improvement of a rising number of flow equipments that associate the manipulation of liquid flow in distinct geometries. The MHD flow of an incompressible, viscous liquid in the micro-channel has been the subject of numerous previous researches due to its potential relevance in several industrial processes. Bejan [1] proposed the second law of thermodynamics aspects of heat transfer by forced convection in terms of four elementary flow structures. Bejan [2] discussed the heat transfer augmentation methods, heat exchanger design, and thermal insulation systems. Abbassi et al. [3] studied the variations of entropy generation and Bejan number as a function Rayleigh number and irreversibility distribution ratio. They found that the entropy generation is higher near the channel walls than that in the central region of the channel. Makinde and Osalusi [4] informed that the heat transfer irreversibility depends on the fluid friction irreversibility and viscous dissipation has no effect on the entropy generation rate near the central region of the channel. Mahmud and Fraser [5] presented the velocity, entropy generation rate, and Bejan numbers variation as a function of Darcy number. Taghizadeh and Asaditaheri [6] observed the maximum Bejan number at high Richardson numbers. Afridi and Qasim [7] informed that the entropy generation rate can be minimized either by increasing the velocity of the needle or by decreasing the free stream velocity. Cimpean and Pop [8] discussed the depreciation of 
entropy generation of a liquid, between two inclined parallel heated porous plates. Eegunjobi and Makinde [9] informed that an enhancement in both slip parameters produced a flow reversal in the Bejan number. Chinyoka et al. [10] presented the entropy generation analysis of an unsteady MHD flow through a porous pipe in the presence of buoyancy effects. Das and Jana [11] studied the entropy generation due to MHD flow in a porous channel in the presence of Navier slip. Hunt et al. [12] presented the heat transfer and classical entropy generation in microreactors, the influences of exothermicity and asymmetry. Das et al. [13] presented the united effects of magnetic field, hydrodynamic slips, and asymmetric convective cooling on the entropy generation in a parallel plate channel under a constant pressure gradient.

In real-life applications several materials like shampoos, paints, condensed milk, muds, printing ink, and tomato paste, etc., show different properties that cannot be easily accepted by the Newtonian theory. So, to characterize such kind of liquids it is required to introduce the concept of non-Newtonian fluid. In 1995, Casson fluid model was developed by Casson for viscoelastic fluid flow. Haq et al. [14] examined the MHD boundary layer flow of a Casson nanofluid with convective boundary condition in the presence of suction or injection. Poornima et al. [15] discussed the radiation and chemical reaction effects on Casson non-Newtonian liquid in the presence of thermal and Navier slip conditions towards a stretching surface. Aboalbashari et al. [16] obtained the entropy generation equation in terms of velocity, temperature, and concentration gradients. Makinde and Eegunjobi [17] observed that the influence of magnetic field and Casson fluid parameter have significant reaction on the entropy generation rate. Gnaneswara Reddy et al. [18] analyzed the hydromagnetic flow of Casson nanofluid towards a cylinder with first order velocity, thermal, and concentration Biot conditions. Shashikumar et al. [19] informed that the entropy generation rate increases with an increase in radiation parameter and Biot number. Gireesha et al. [20] presented the entropy generation and heat transport analysis of Casson fluid flow in the presence of viscous and Joule heating in an inclined micro-porous-channel. Kalyan Kumar and Srinivas [21] presented the influence of joule heating and thermal radiation on unsteady hydromagnetic flow of chemically reacting Casson fluid over an inclined porous stretching sheet.

MHD pumps are already in use in chemical energy technology for pumping electrically conducting fluids at some of the atomic energy centers. Besides these applications, when the fluid is electrically conducting, the natural convection flow is appreciably influenced by an imposed magnetic field. In the past years, several MHD natural convection solutions were obtained by Venkateswarlu et al. [22-27]. Manjunatha et al. [28] discussed the Peristaltic flow of a Jeffery fluid over a porous conduit in the presence of variable liquid properties and convective boundary conditions. Goud et al. [29] presented the mass transfer effects on MHD flow through porous medium past an exponentially accelerated inclined plate with variable temperature and thermal radiation. Manjunatha et al. [30] performed the heat transfer analysis on peristaltic transport of a Jeffery fluid in an inclined elastic tube with porous walls. Nayak et al. [31] studied the influence of non-uniform heat source/sink and variable viscosity on mixed convection flow of third grade nanofluid over an inclined stretched riga plate.

In this article, our goal is to present the united effects of Reynolds number, heat generation, hydrodynamic slips, and convective boundary conditions on entropy generation rate and Bejan number in an MHD Casson fluid flow through a parallel plate channel with a constant pressure gradient. There is no such study by any former researchers on the steady hydromagnetic Casson fluid flow in a parallel plate channel in the presence of heat generation. The present study is an extension of the idea of Ibanez [32] by including the Casson fluid parameter and heat generation parameter. 


\section{Formulation of the problem}

A fully developed steady hydromagnetic flow of Casson fluid through a microchannel with two horizontal parallel porous plates separated by a distance $a$ in the presence of Joule heating, viscous dissipation, constant longitudinal pressure gradient $d p / d x$ have been studied. A schematic view of the hydromagnetic micro-channel under investigation is displayed in Fig. 1. A uniform transverse magnetic field of strength $B_{0}$ is applied perpendicular to the porous plates. Select the Cartesian coordinate system with $x$-axis aligned at the center of the micro-channel in the direction of the flow and the $y$-axis normal to the porous plates. The plates are of infinite length in $x$-direction so that the physical quantities are independent on $x$. Hence, all the physical quantities are depending on $y$ only. The lower plate is located at $y=0$ and the upper plate of the channel is located at $y=a, y$ represents the transversal coordinate. Convective boundary conditions are applied for the solution of the heat transfer equation. We assume that the velocity satisfies the slip condition at the plates for the solution of the momentum equation. Under the expected Boussinesq's approximation the governing momentum, thermal energy and entropy generation equations are (see, Guillermo [32]):

Continuity equation:

$$
\frac{d v}{d y}=0
$$

Momentum equation:

$V_{0} \frac{d u}{d y}=-\frac{1}{\rho} \frac{d p}{d x}+v\left(1+\frac{1}{\beta}\right) \frac{d^{2} u}{d y^{2}}-\frac{\sigma B_{0}^{2}}{\rho} u-\frac{v}{k} u$

Energy equation:

$V_{0} \frac{d T}{d y}=\frac{k_{T}}{\rho c_{p}} \frac{d^{2} T}{d y^{2}}+\frac{v}{c_{p}}\left(1+\frac{1}{\beta}\right)\left[\frac{d u}{d y}\right]^{2}+\frac{\sigma B_{0}^{2}}{\rho c_{p}} u^{2}+\frac{v}{k c_{p}} u^{2}+\frac{Q_{0}}{\rho c_{p}}\left(T-T_{a}\right)$

Entropy generation equation:

$$
E_{g}=\frac{v \rho}{T_{a}}\left(1+\frac{1}{\beta}\right)\left[\frac{d u}{d y}\right]^{2}+\frac{\sigma B_{0}^{2}}{T_{a}} u^{2}+\frac{1}{T_{a}} \frac{v \rho}{k} u^{2}+\frac{k_{T}}{T_{a}^{2}}\left[\frac{d T}{d y}\right]^{2}
$$

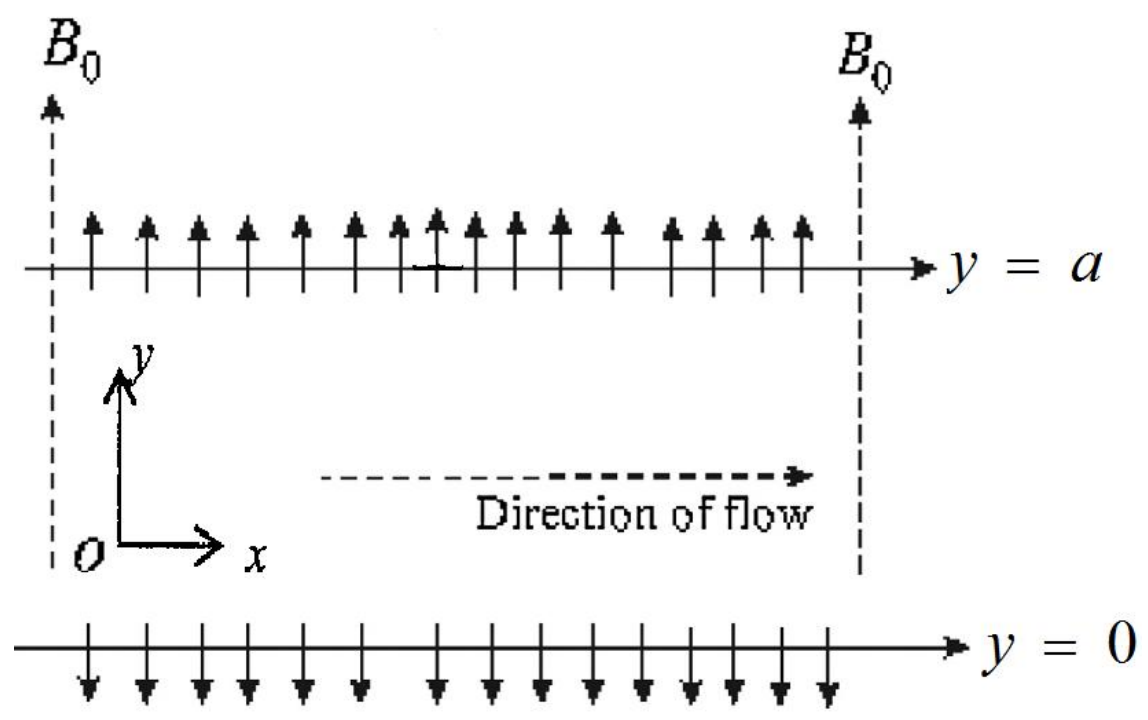

Fig.1: Flow formation and coordinate system. 
Here $u$-fluid velocity in $x$-direction, $v$-fluid velocity in $y$-direction, $V_{0}$ - uniform suction/injection velocity, $\rho$ - density, $v$ - kinematic viscosity, $\beta$-Casson parameter, $\sigma-$ electrical conductivity, $B_{0}$ - uniform magnetic field, $k$-permeability of the porous medium, $T$ - fluid temperature, $k_{T}$ - thermal conductivity, $c_{p}$ - specific heat, $Q_{0}$ - heat source coefficient, $T_{a}$-ambient temperature and $E g$-entropy generation respectively.

The first term on right-hand side of equation (4) represents the entropy generation due to viscous dissipation, the second term represents the entropy generation due to heat transfer irreversibility, the third term represents the entropy generation due to the magnetic field and the fourth term represents the entropy generation due to porous medium respectively.

We should in prior inform the reviewer that our mathematical model is not the same as that by Guillermo [32] in which the Casson parameter, permeability of porous medium and heat source coefficient were not taken into account. The initial and boundary conditions for the velocity and temperature fields are

$$
\begin{aligned}
& u+\alpha_{1} \frac{d u}{d y}=0, \quad \frac{d T}{d y}=\frac{h_{1}\left(T_{a}-T\right)}{k_{T}} \quad \text { at } \quad y=a \\
& u-\alpha_{2} \frac{d u}{d y}=0, \quad \frac{d T}{d y}=\frac{h_{2}\left(T-T_{h}\right)}{k_{T}} \quad \text { at } \quad y=0
\end{aligned}
$$

Here $\alpha_{1}$-slip length of the upper plate, $\alpha_{2}$ - slip length of the lower plate, $h_{1}-$ convective heat transfer coefficient of the upper plate, $h_{2}$ - convective heat transfer coefficient of the lower plate, $T_{h}$ - hot fluid temperature and $a$ - distance between two plates respectively.

We introducing the following dimensionless quantities:

$$
U=\frac{a}{v} u, \eta=\frac{y}{a}, \beta_{1}=\frac{\alpha_{1}}{a}, \beta_{2}=\frac{\alpha_{2}}{a}, \theta=\frac{T-T_{a}}{T_{h}-T_{a}}
$$

Using equation (7) in equations (2), (3), and (4) we obtain the non-dimensional equations as

$$
\begin{aligned}
& \operatorname{Re} \frac{d U}{d \eta}=P+\left(1+\frac{1}{\beta}\right) \frac{d^{2} U}{d \eta^{2}}-\left(M+\frac{1}{K}\right) U \\
& P e \frac{d \theta}{d \eta}=\frac{d^{2} \theta}{d \eta^{2}}+E c \operatorname{Pr}\left[\left(1+\frac{1}{\beta}\right)\left[\frac{d U}{d \eta}\right]^{2}+\left(M+\frac{1}{K}\right) U^{2}\right]+H \theta \\
& N_{S}=\frac{E_{g}}{E_{g 0}}=E c \operatorname{Pr} W\left[\left(1+\frac{1}{\beta}\right)\left[\frac{d U}{d \eta}\right]^{2}+\left(M+\frac{1}{K}\right) U^{2}\right]+\left[\frac{d \theta}{d \eta}\right]^{2}
\end{aligned}
$$

Here $R e=\frac{V_{0} a}{v}$ is the Reynolds number, $P=-\frac{a^{3}}{\rho v^{2}} \frac{d p}{d x}$ is the pressure gradient parameter, $M=\frac{\sigma B_{0}^{2} a^{2}}{v \rho}$ is the Hartmann number, $K=\frac{k}{a^{2}}$ is the permeability parameter, $\operatorname{Pr}=\frac{v \rho c_{p}}{k_{T}}$ is the Prandtl number, $\operatorname{Pe}=\operatorname{Re} \operatorname{Pr}$ is the Peclet number, $E c=\frac{v^{2}}{a^{2}\left(T_{h}-T_{a}\right) c_{p}}$ is the Eckert number, $H=\frac{Q_{0} a^{2}}{k_{T}}$ is the heat generation parameter, $E g_{0}=\frac{k_{T}\left(T_{h}-T_{a}\right)^{2}}{a^{2} T_{a}^{2}}$ is the 
characteristic entropy generation rate, $W=\frac{T_{a}}{T_{h}-T_{a}}$ is the characteristic temperature ratio respectively. given by

The corresponding initial and boundary conditions in dimensionless quantities are

$$
\begin{array}{ll}
U+\beta_{1} \frac{d U}{d \eta}=0, \frac{d \theta}{d \eta}+B_{1} \theta=0 & \text { at } \eta=1 \\
U-\beta_{2} \frac{d U}{d \eta}=0, \frac{d \theta}{d \eta}+B_{2}(1-\theta)=0 & \text { at } \eta=0
\end{array}
$$

Here $B_{1}=\frac{a h_{1}}{k_{T}}$ is the Biot number for upper plate and $B_{2}=\frac{a h_{2}}{k_{T}}$ is the Biot number for lower plate respectively.

The Bejan number $B e$ can be expressed as

$$
B e=\frac{H T I}{N S}=\frac{H T I}{F F I+H T I}
$$

Here $\quad F F I=E c \operatorname{Pr} W\left[\left(1+\frac{1}{\beta}\right)\left[\frac{d U}{d \eta}\right]^{2}+\left(M+\frac{1}{K}\right) U^{2}\right] \quad$ is $\quad$ the dissipative irreversibility, $H T I=\left[\frac{d \theta}{d \eta}\right]^{2}$ is the heat transfer irreversibility and $N s=F F I+H T I$ is the total entropy generation respectively.

The Bejan number $B e$ assumes the value between 0 and 1 . The value $B e=0$ coincide to the case when irreversibility is dominated by the fluid friction effects and $B e=1$ coincide to the case when irreversibility due to the heat transfer by temperature inequality has taken its toll.

It is now important to calculate physical quantities of our primary interest, which are the skin friction coefficient and heat transfer coefficient. Given the micro-channel slip velocity and temperature fields in the boundary layer, shear stress $\tau_{w}$, and heat flux $q_{w}$ are obtained as

$$
\begin{aligned}
\tau_{w} & =v \frac{d u}{d y} \\
q_{w} & =-k_{T} \frac{d T}{d y}
\end{aligned}
$$

In non-dimensional form the skin-friction coefficient $\tau$ and heat transfer coefficient $\mathrm{Nu}$ are defined as

$$
\begin{aligned}
& \tau=\frac{a^{2}}{v^{2}} \tau_{w} \\
& N u=\frac{a}{k_{T}\left(T_{h}-T_{a}\right)} q_{w}
\end{aligned}
$$

Using the non-dimensional quantities in equation (7) and the equations (14) to (15) into equations (16) to (17), we obtain the physical parameters

$$
\tau=\frac{d U}{d \eta}
$$


$N u=-\frac{d \theta}{d \eta}$

\section{Solution of the problem}

We solve the non-dimensional equations (8) and (9) analytically with initial and boundary conditions in equations (11) and (12). Equation (8) has the following analytical solution:

$$
U(\eta)=a_{8} \exp \left(m_{1} \eta\right)+a_{9} \exp \left(m_{2} \eta\right)+a_{7}
$$

When the velocity field is obtained, the temperature field for the liquid is determined by the solution of the boundary value problem in equation (9), with corresponding boundary conditions in equations (11) and (12). The analytical expression for the liquid temperature profile is

$$
\begin{aligned}
& \theta(\eta)=a_{32} \exp \left(m_{3} \eta\right)+a_{33} \exp \left(m_{4} \eta\right)-a_{19} \exp \left(m_{1} \eta\right)-a_{18} \exp \left(m_{2} \eta\right)- \\
& a_{15} \exp \left(2 m_{1} \eta\right)-a_{16} \exp \left(2 m_{2} \eta\right)-a_{17} \exp \left(\left(m_{1}+m_{2}\right) \eta\right)
\end{aligned}
$$

3.1. Heat transfer coefficient: From the temperature of the liquid, the heat transfer coefficient can be expressed as

$$
\begin{aligned}
& N u(\eta)=-\left[\begin{array}{l}
a_{32} m_{3} \exp \left(m_{3} \eta\right)+a_{33} m_{4} \exp \left(m_{4} \eta\right)-a_{19} m_{1} \exp \left(m_{1} \eta\right)-a_{18} m_{2} \exp \left(m_{2} \eta\right)- \\
2 a_{15} m_{1} \exp \left(2 m_{1} \eta\right)-2 a_{16} m_{2} \exp \left(2 m_{2} \eta\right)-a_{17}\left(m_{1}+m_{2}\right) \exp \left(\left(m_{1}+m_{2}\right) \eta\right)
\end{array}\right] \\
& N u(0)=-\left[a_{32} m_{3}+a_{33} m_{4}-a_{19} m_{1}-a_{18} m_{2}-2 a_{15} m_{1}-2 a_{16} m_{2}-a_{17}\left(m_{1}+m_{2}\right)\right] \\
& N u(1)=-\left[\begin{array}{l}
a_{32} m_{3} \exp \left(m_{3}\right)+a_{33} m_{4} \exp \left(m_{4}\right)-a_{19} m_{1} \exp \left(m_{1}\right)-a_{18} m_{2} \exp \left(m_{2}\right)- \\
2 a_{15} m_{1} \exp \left(2 m_{1}\right)-2 a_{16} m_{2} \exp \left(2 m_{2}\right)-a_{17}\left(m_{1}+m_{2}\right) \exp \left(m_{1}+m_{2}\right)
\end{array}\right]
\end{aligned}
$$

3.2. Skin friction coefficient: From the micro-channel slip velocity, the skin friction coefficient can be expressed as

$$
\begin{aligned}
& \tau(\eta)=m_{1} a_{8} \exp \left(m_{1} \eta\right)+m_{2} a_{9} \exp \left(m_{2} \eta\right) \\
& \tau(0)=m_{1} a_{8}+m_{2} a_{9} \\
& \tau(1)=m_{1} a_{8} \exp \left(m_{1}\right)+m_{2} a_{9} \exp \left(m_{2}\right)
\end{aligned}
$$

\section{Results and discussion}

The parameters that impact this flow are the Reynolds number $R e$, Casson parameter $\beta$, Hartmann number $M$, permeability parameter $K$, pressure gradient parameter $P$, upper wall slip parameter $\beta_{1}$, lower wall slip parameter $\beta_{2}$, Peclet number $P e$, Eckert number $E c$, Prandtl number $\mathrm{Pr}$, heat generation parameter $H$, Biot number for upper plate $B_{1}$, and Biot number for lower plate $B_{2}$. To review the impact of these parameters, the deviation in velocity, temperature, entropy generation, Bean number, skin-friction and rate of heat transfer are relevant in Figs. 2-14 as well as tables 1-9. The parametric study has been performed with fixed values of $R e=1, P=1, \beta=0.1, M=1, K=1, P e=1, E c=1, H=1$, $B_{1}=1.5, B_{2}=1.5, \operatorname{Pr}=0.71, w=1, \beta_{1}=1.5$ and $\beta_{2}=1.5$.

It is seen from the Table 1 that the skin-friction coefficient enhances at both walls with an increase in the Casson parameter and permeability parameter. Table 2 shows that the skin friction coefficient at the lower wall decreases with an increase in the Reynolds number 
and Hartmann number. The skin friction coefficient at the upper wall increases with an increase in the Reynolds number whereas it decreases with an increase in the Hartmann number. It is pointed out from the Table 3 that the skin friction coefficient declines at both walls with an increase in the upper wall slip parameter and lower wall slip parameter.

It is noticed from the Table 4 that the heat transfer coefficient at both walls declines with an increase in the values of Casson parameter whereas it increases at both walls with an increase in the value of permeability parameter. Table 5 shows that the heat transfer coefficient at both walls decreases with an increase in the values of Reynolds number and Hartmann number. It is observed from the Table 6 that the heat transfer coefficient at the lower wall enhances with an increase in the values of upper wall slip parameter and lower wall slip parameter. The rate of heat transfer at the upper wall decreases with an increase in the upper wall slip parameter whereas it increases with an increase in the lower wall slip parameter. It is pointed out from the Table 7 that the heat transfer coefficient at the lower wall increases with an increase in the values of Peclet number and Eckert number. The heat transfer coefficient at the upper wall declines with an increase in the value of Peclet number whereas it increases with an increase in the value of Eckert number. It is noticed from the Table 8 that the heat transfer coefficient at both walls decreases with an increase in the values of Prandtl number and heat generation parameter. It is seen from the Table 9 that the heat transfer coefficient at the lower wall increases with an increase in the values of Biot number for upper wall and Biot number for lower wall. The heat transfer coefficient at the upper wall increases with an increase in the value of Biot number for upper wall whereas it decreases with an increase in the value of Biot number for lower wall.

Figs. 2(a)-(d) demonstrates the impact of Reynolds number on velocity, temperature, entropy generation rate, and Bejan number. It is obvious from Figure 2(a) that as Reynolds number rises, the fluid velocity declines. It is seen from Figure 2(b) that, enhancement in the value of the Reynolds number leads to an increase in the temperature of the liquid. It is manifest from Figures 2(c)-(d) that an enhancement in Reynolds number leads to growth in entropy generation and Bejan number near the lower wall and decreases toward the upper wall.

The effects of Casson parameter on the velocity, temperature, entropy generation, and Bejan number profiles are displayed in Figure 3(a)-(d). It is seen from figure 3(a) that the greater values of Casson parameter leads to a reduction near the lower wall and an enhancement near the upper wall in the fluid velocity profile. Figure 3(b)-(d) elucidates the increasing behavior of temperature, entropy generation, and Bejan number profiles for rising values of Casson parameter.

The velocity, temperature, entropy generation, and Bejan number changes for distinct values of the Hartmann number are exhibited in Figure 4(a)-(d). It is obvious from Figure 2(a) that as Hartmann number rises, the fluid velocity declines. The basis of such a diminishing scenario is the Lorentz influence which produces a retardation force beside the magnetic field. So, velocity diminishes. It reveals from Figure 4(b) that the temperature of the liquid within the channel, increasing in the presence of Hartmann number. It is manifest from Figure 4(c)(d) that an enhancement in Hartmann number leads to growth in entropy generation and Bejan number near the lower wall and decreases toward the upper wall.

In Figure 5(a)-(d), the velocity, temperature, entropy generation, and Bejan number variations for different values of permeability parameter are shown, respectively. It is noticed from Figure 5(a) that velocity increases with an increase in the permeability parameter. One can observed from Figure 5(b) that as the temperature profile decreases with the permeability parameter increases. Escalating value of permeability parameter produces more entropy 
generation rate as displayed in Figure 5(c). In Figure 5(d), Bejan number decreases near the lower wall and it increases near the upper wall for rising values of permeability parameter.

The variations of velocity, temperature, entropy generation, and Bejan number for the effect of pressure gradient parameter are as displayed in Figure 6(a)-(d). It reveals from Figure 6(a)-(c) that the velocity and entropy generation rate of the liquid, increasing in the presence of pressure gradient parameter and reverse phenomenon occurs for the temperature field. Figure 6(d) reveals that a rise in the Beajan number is observed with an enhancement in pressure gradient parameter at the upper wall, but the Beajan number decreases due to increasing pressure gradient parameter at the lower wall.

The influences of velocity, temperature, entropy generation, and Bejan number for the effect of upper wall slip length parameter are as displayed in Figure 7(a)-(d). It reveals from Figure 7(a)-(c) that the velocity and entropy generation rate of the liquid, decreasing in the presence of upper wall slip length parameter and reverse phenomenon occurs for the temperature field. Figure 7(d) reveals that a rise in the Beajan number is observed with an enhancement in upper wall slip length parameter at the lower wall, but the Beajan number decreases due to increasing upper wall slip length parameter at the upper wall.

The velocity, temperature, entropy generation, and Bejan number changes for distinct values of the lower wall slip length parameter are exhibited in Figure 8(a)-(d). It is obvious from Figure 8(a)-(c) that as lower wall slip length parameter rises, the liquid velocity and entropy generation rate accelerates and temperature field declines. It is manifest from Figure 8(d) that an enhancement in the lower wall slip length parameter leads to growth in Bejan number near the upper wall and decreases near the lower wall.

In Figure 9(a)-(c), the temperature, entropy generation rate, and Bejan number profiles for different values of Peclet number are shown, respectively. It is obvious from Figure 9(a) that as Peclet number rises, the liquid temperature declines. It reveals from Figure 9(b)-(c) that the entropy generation rate and Bejan number of the liquid, decreases near the walls of the micro-channel and increases near the middle portion of the micro-channel in the presence of Peclet number.

Figure 10(a)-(c) reveals the temperature, entropy generation rate, and Bejan number profiles for various values of the Eckert number. It is noticed from Figure 10(a)-(b) that an enhancement in the Eckert number leads to a reduction in the liquid temperature and an enhancement in the entropy generation rate. From Figure 10(c), reverse phenomenon can be seen near the walls of the micro-channel. It is noticed that the Bejan number diminishes near the lower wall and rises near the upper wall as Eckert number enhances.

The influences of the Prandtl number on the temperature, entropy generation rate, and Bejan number profiles are demonstrated in Figure 11(a)-(c). From Figure 11(a), it is obvious that as the Prandtl number rises, there is a decline in the liquid temperature. Physically, the enhancement in the Prandtl number reduces the temperature within the flow, and by which weak convection current within the flow regime. From Figure 11(b), we have seen that the entropy generation rate in the presence of the Prandtl number is enhanced. In Figure 11(c), it is noticed that the Bejan number diminishes near the lower wall and rises near the upper wall as Prandtl number enhances.

The influences of the heat the generation parameter on the temperature, entropy generation rate, and Bejan number profiles have been illustrated in Figure 12(a)-(c). It can be noticed that the liquid temperature, entropy generation rate, and Bejan number profiles are increases with an increase in the heat generation parameter. 
Figure 13(a) depicts that the liquid temperature decreases with an increase in the Biot number for upper plate. Figure 13(b)-(c) reveals that a rise in the entropy generation rate and Bejan number is observed with an enhancement in the Biot number for upper plate near the upper wall, but the entropy generation rate and Bejan number decreases due to increasing the Biot number for upper plate near the lower wall.

Figure 14(a) depicts that the liquid temperature decreases with an increase in the Biot number for lower plate. Figure 13(b)-(c) reveals that a rise in the entropy generation rate and Bejan number is observed with an enhancement in the Biot number for lower plate near the upper wall, but the entropy generation rate and Bejan number decreases due to increasing the Biot number for lower plate near the lower wall.

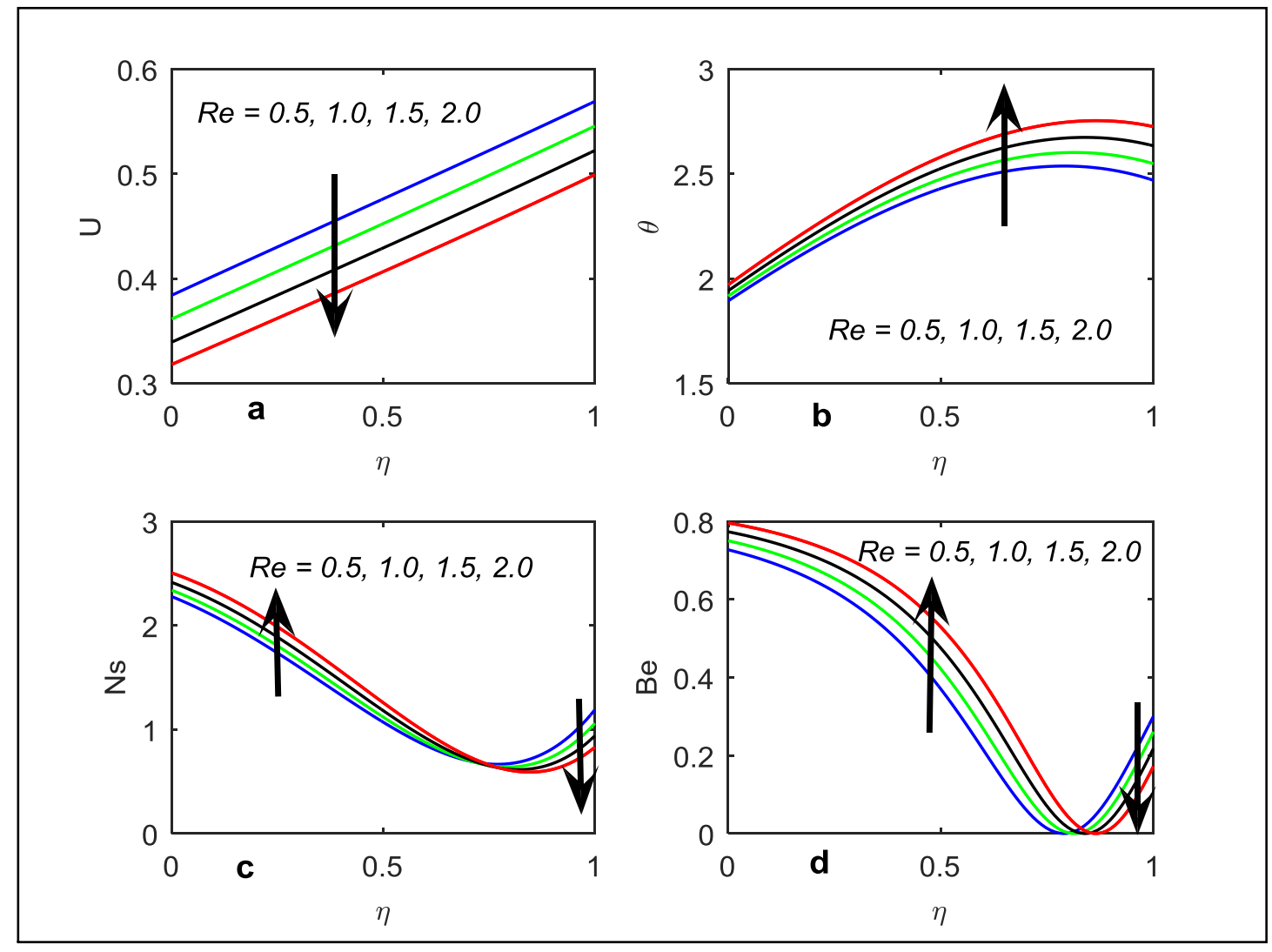

Fig. 2 Impact of $\operatorname{Re}$ on $U, \theta, N s$ and $B e$. 


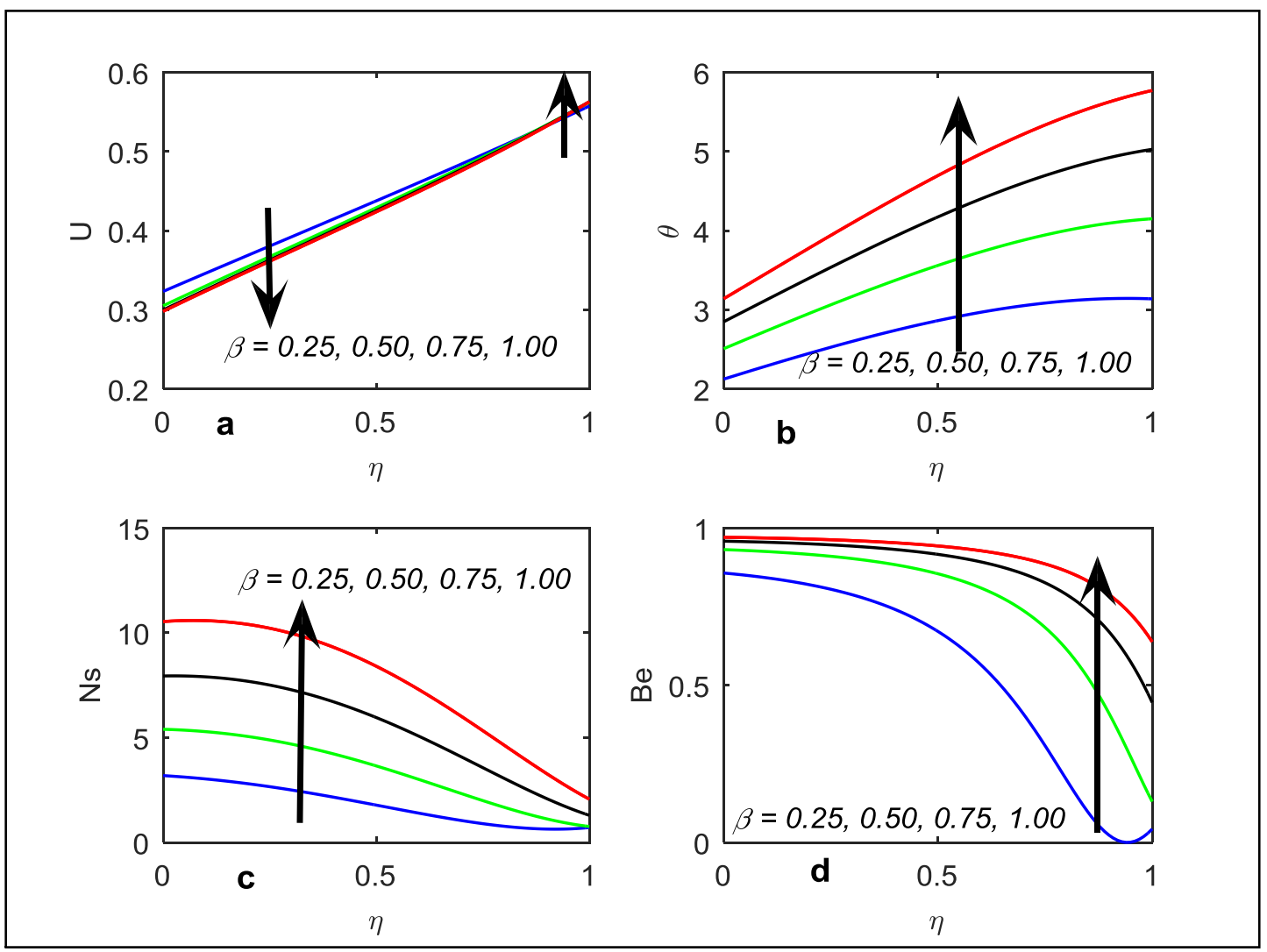

Fig. 3 Impact of $\beta$ on $U, \theta, N s$ and $B e$.

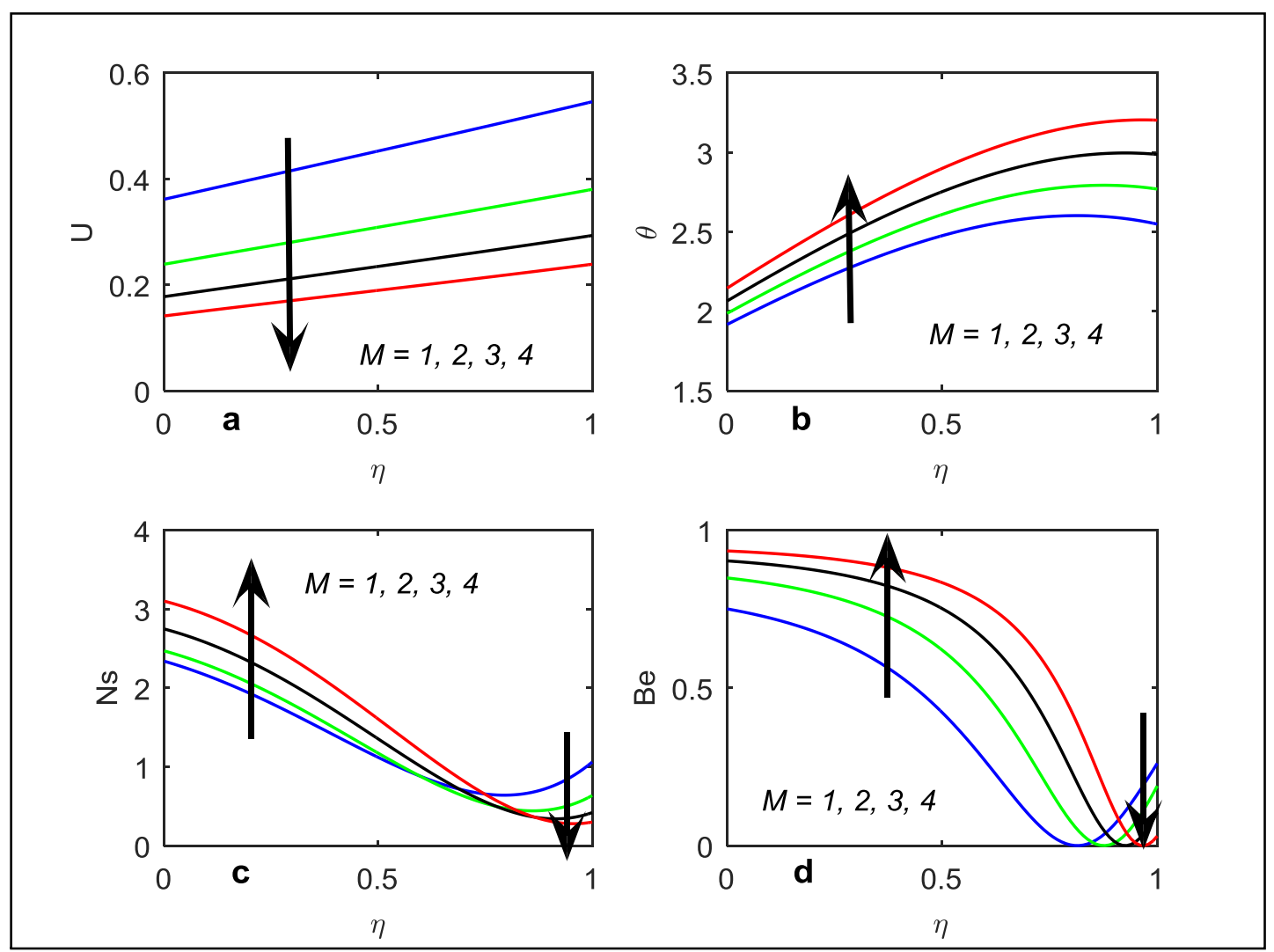

Fig. 4 Impact of $M$ on $U, \theta, N s$ and $B e$. 

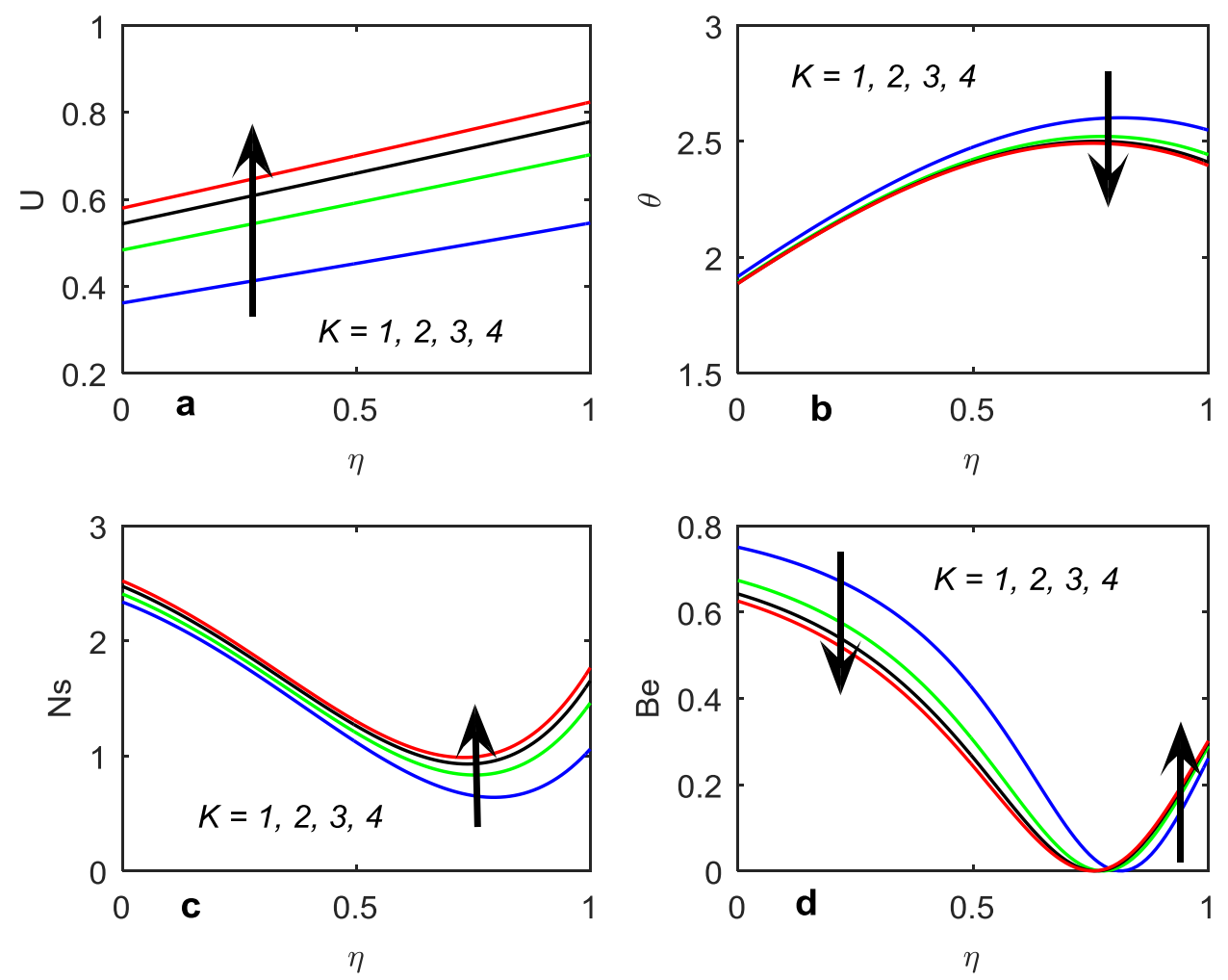

Fig. 5 Impact of $K$ on $U, \theta, N s$ and $B e$.
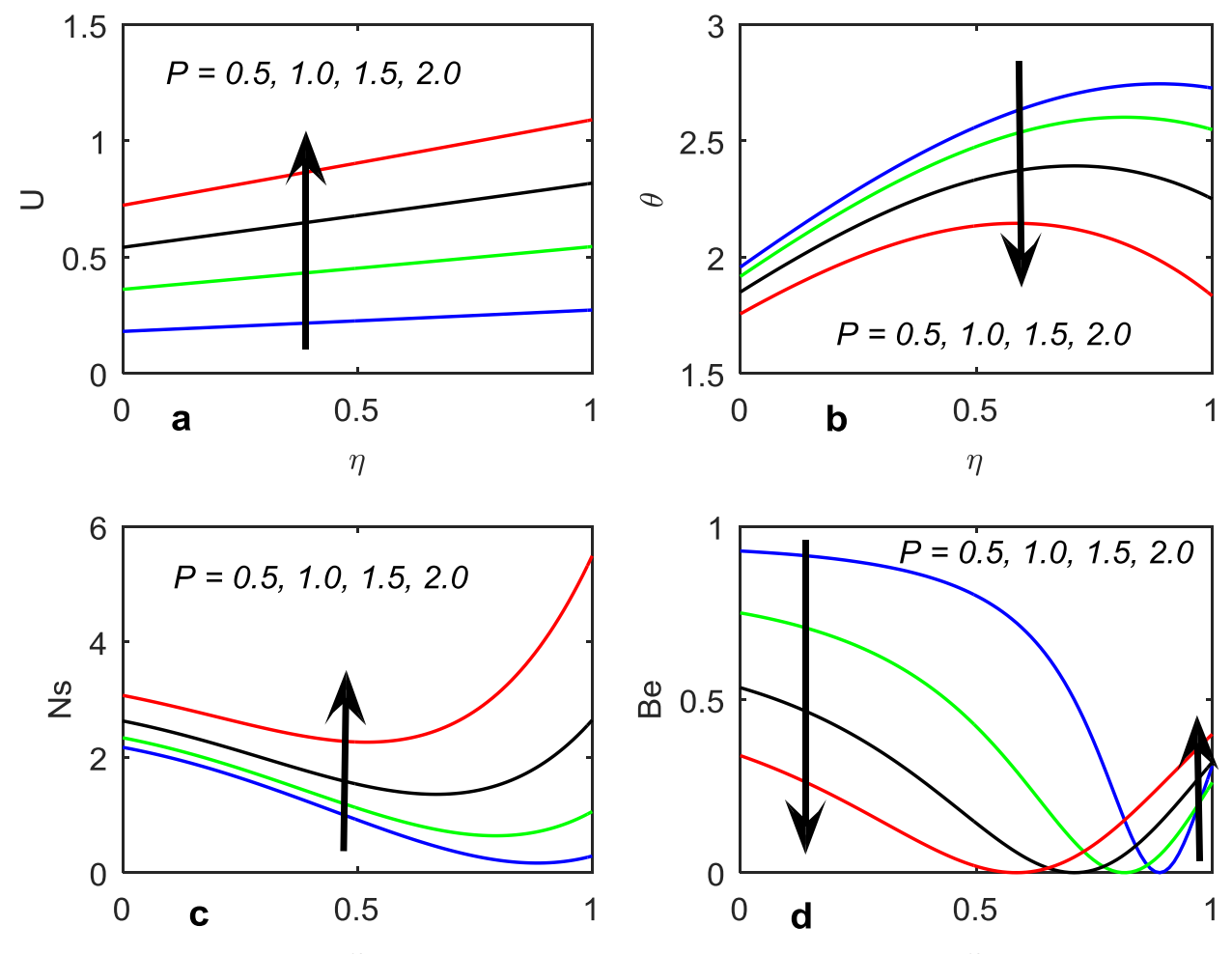

Fig. 6 Impact of $P$ on $U, \theta, N s$ and $B e$. 


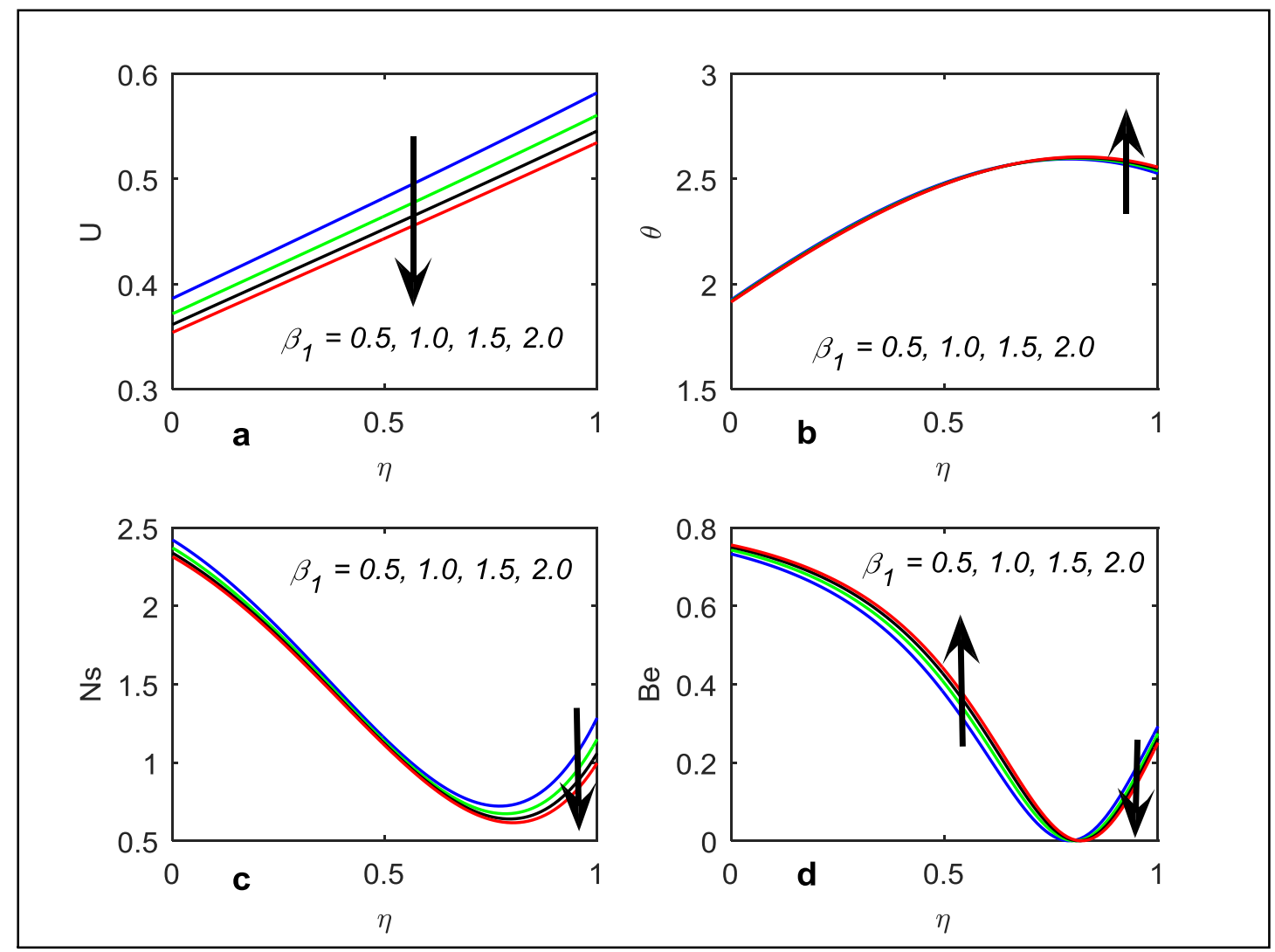

Fig. 7 Impact of $\beta_{1}$ on $U, \theta, N s$ and $B e$.
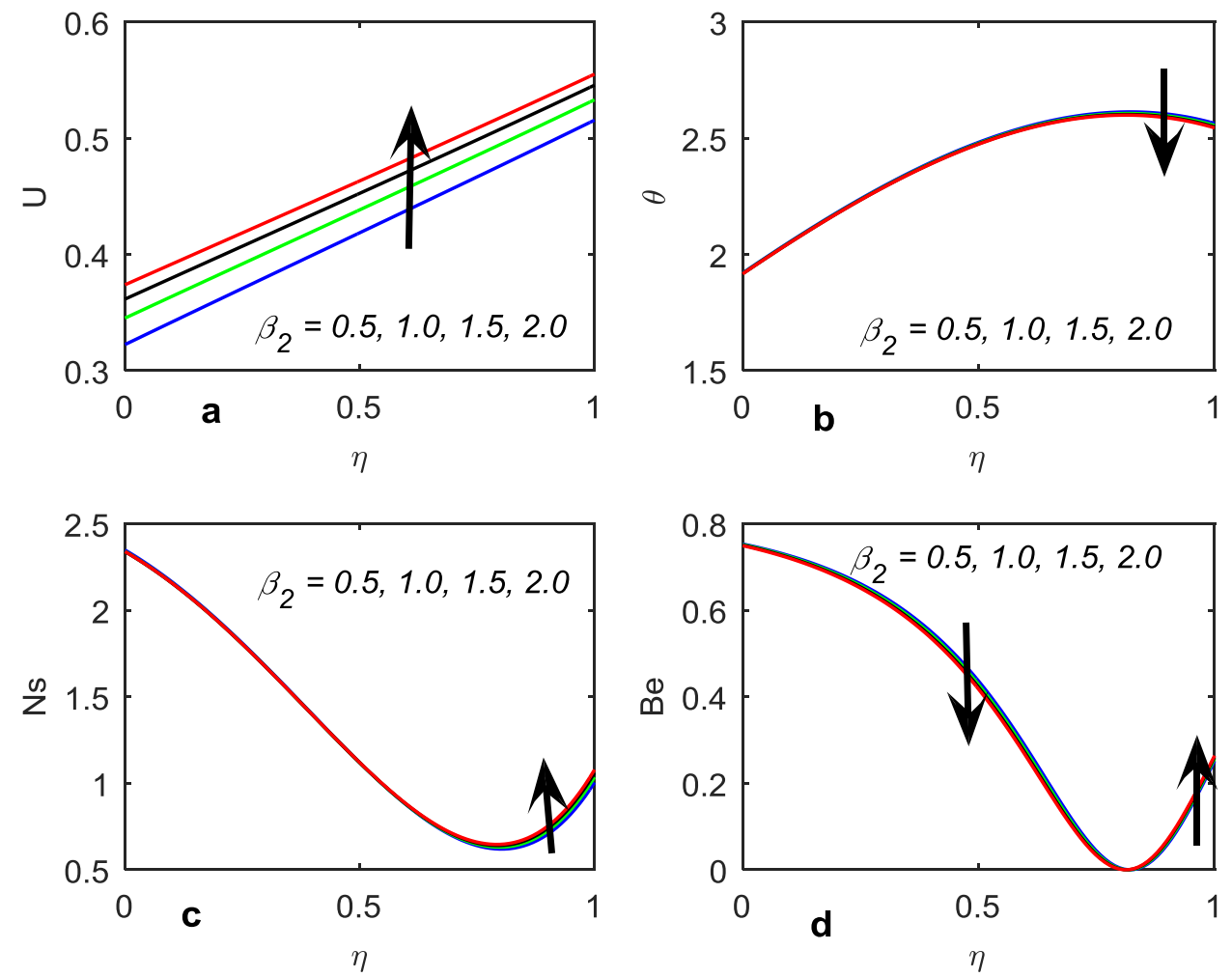

Fig. 8 Impact of $\beta_{2}$ on $U, \theta, N s$ and $B e$. 


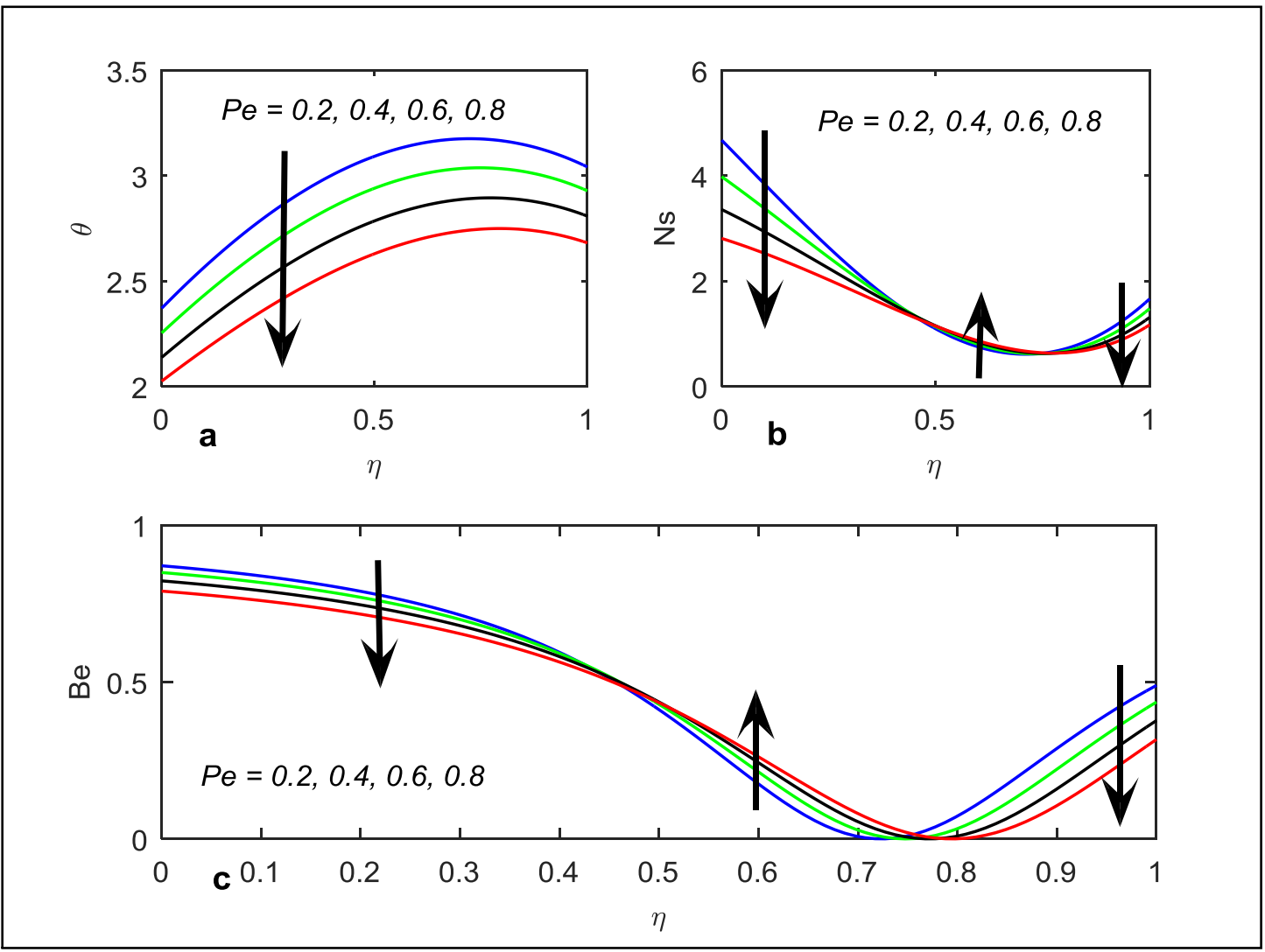

Fig. 9 Impact of $P e$ on $\theta, N s$ and $B e$.
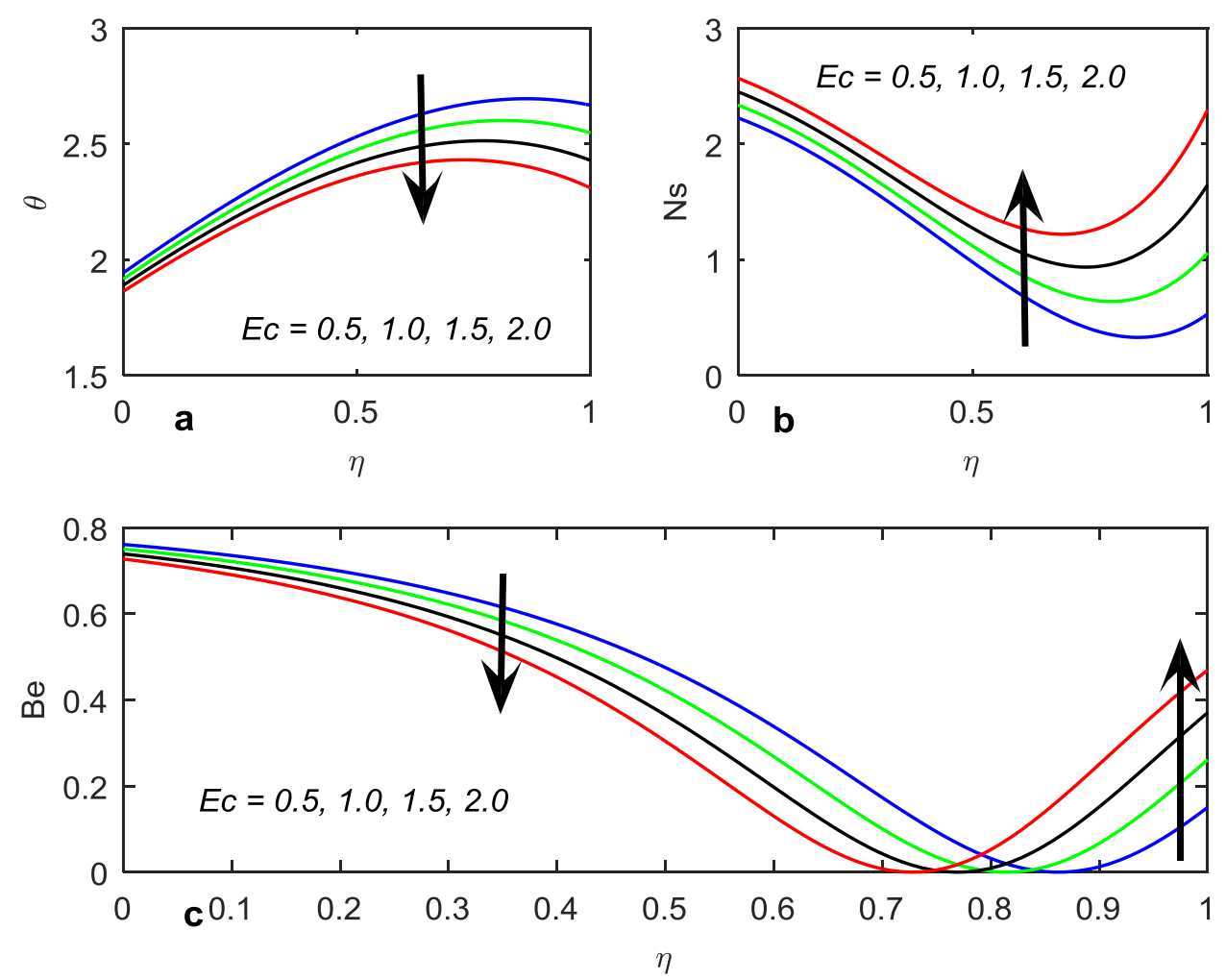

Fig. 10 Impact of $E c$ on $\theta, N s$ and $B e$. 

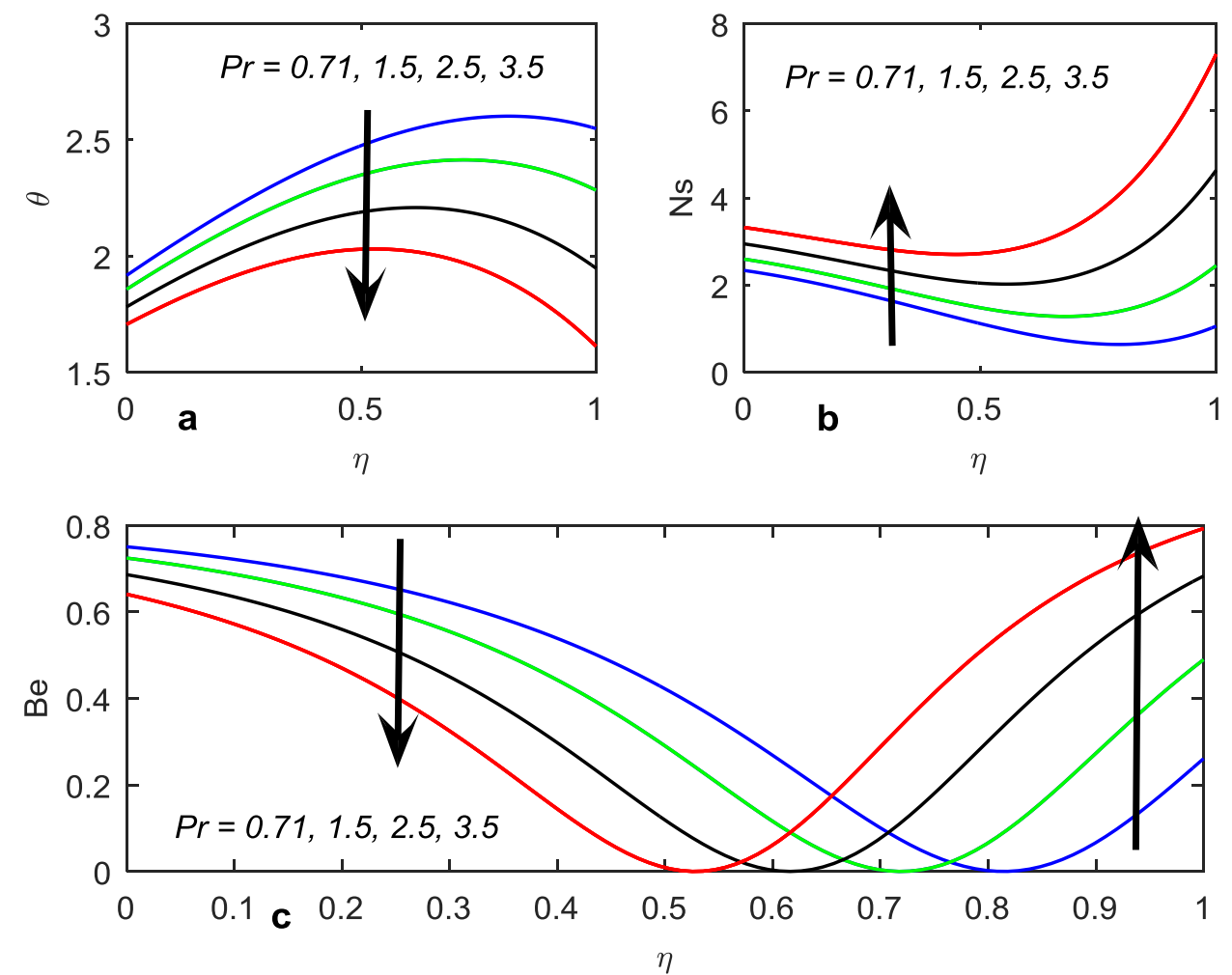

Fig. 11 Impact of $\operatorname{Pr}$ on $\theta, N s$ and $B e$.
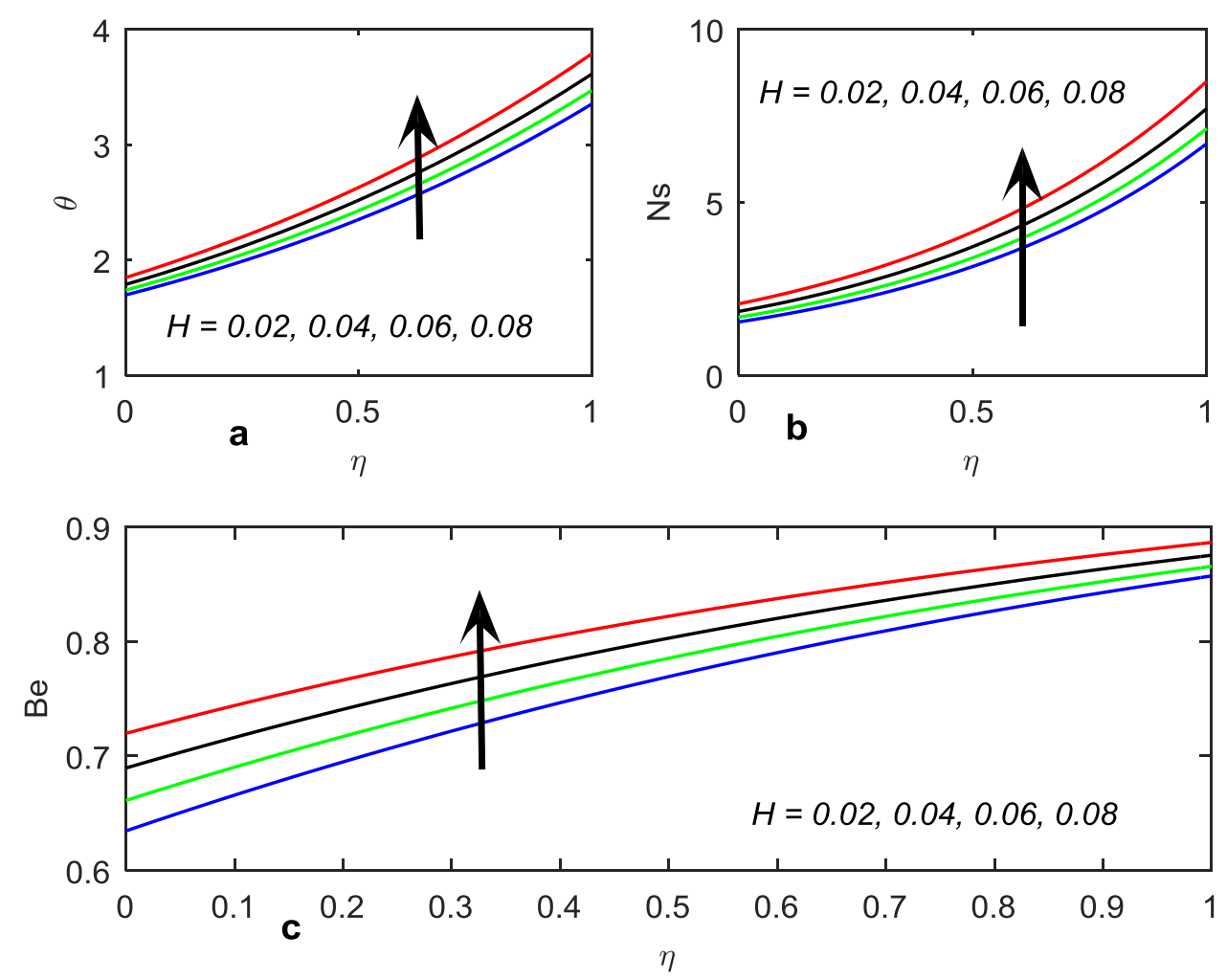

Fig. 12 Impact of $H$ on $\theta, N s$ and $B e$. 

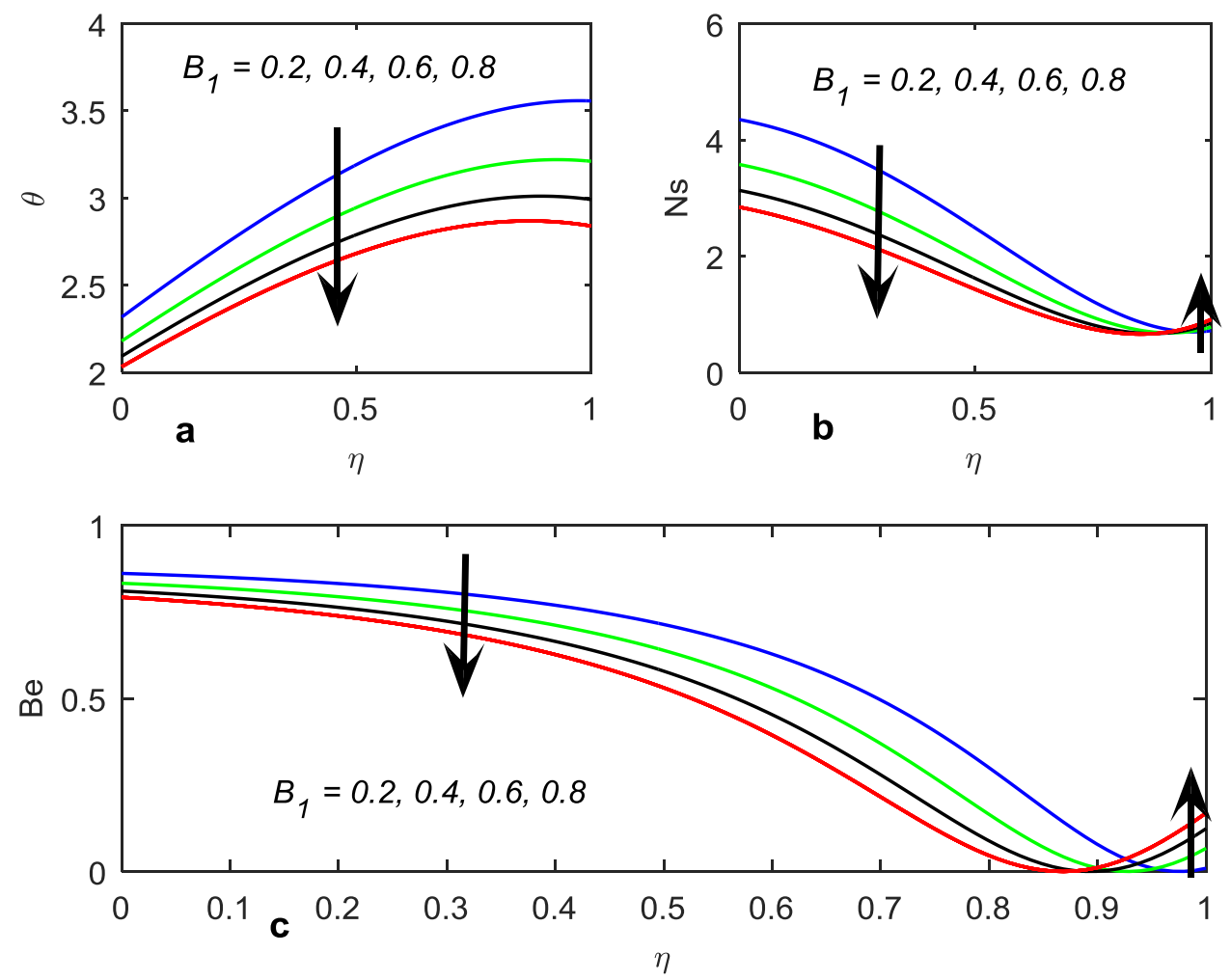

Fig. 13 Impact of $B_{1}$ on $\theta, N s$ and $B e$.
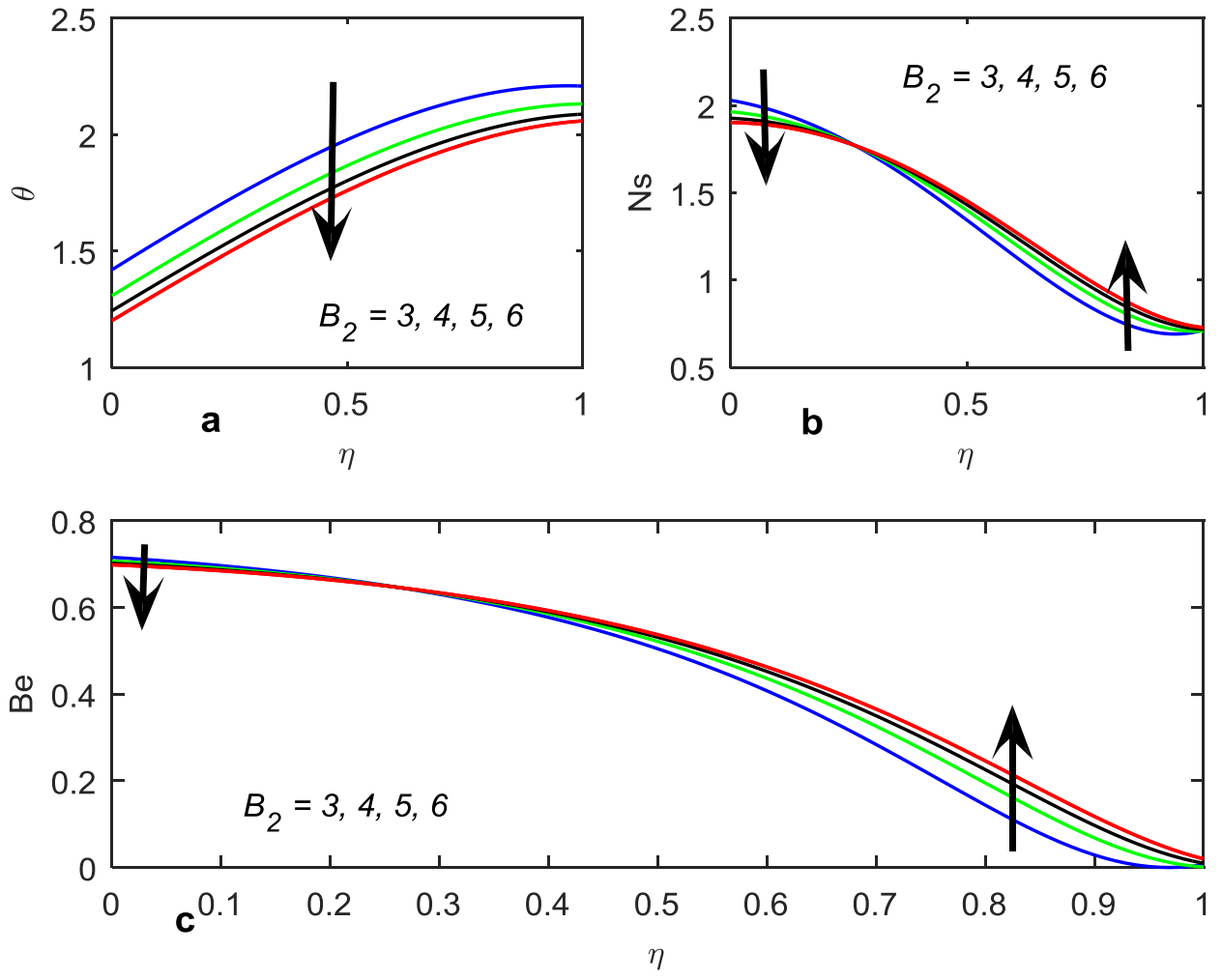

Fig. 14 Impact of $B_{2}$ on $\theta, N s$ and $B e$. 


\section{Conclusions}

Main experiences of present investigation are listed below:

- The fluid velocity reduces near the lower wall and rises near the upper wall as the Casson fluid parameter increases. The increase in the Casson fluid parameter leads to enhance in the temperature, entropy generation rate, and Bejan number. The increase in the Casson fluid parameter leads to enhance in the skin-friction and reduce in the rate of heat transfer.

- The greater values of permeability parameter increase the velocity and entropy generation rate. Temperature profile decreases for rising values of the permeability parameter. As permeability parameter increases the Bejan number decreases near the lower wall and increases near the upper wall. The increase in the permeability parameter leads to enhance in the skin-friction and rate of heat transfer.

- The fluid temperature, entropy generation rate, and Bejan number increases as the heat generation parameter increases. The heat transfer coefficient reduces as the heat generation parameter increases.

Table. 1 Variation of skin-friction with Casson parameter and permeability parameter

\begin{tabular}{|l|l|l|l|l|l|l|l|l|}
\hline \multirow{2}{*}{$\beta / K$} & \multicolumn{4}{|l|}{ Lower plate $(\eta=0)$} & \multicolumn{4}{l|}{ Upper plate $(\eta=1)$} \\
\cline { 2 - 10 } & 1.0 & 2.0 & 3.0 & 4.0 & 1.0 & 2.0 & 3.0 & 4.0 \\
\hline 0.25 & 0.2311 & 0.2810 & 0.3032 & 0.3159 & 0.2534 & 0.3086 & 0.3333 & 0.3473 \\
\hline 0.50 & 0.2525 & 0.3130 & 0.3402 & 0.3557 & 0.2919 & 0.3635 & 0.3958 & 0.4142 \\
\hline 0.75 & 0.2580 & 0.3228 & 0.3522 & 0.3690 & 0.3085 & 0.3890 & 0.4255 & 0.4464 \\
\hline 1.00 & 0.2595 & 0.3265 & 0.3569 & 0.3743 & 0.3177 & 0.4037 & 0.4429 & 0.4653 \\
\hline
\end{tabular}

Table 2 Variation of skin-friction with Reynolds number and Hartmann number

\begin{tabular}{|l|l|l|l|l|l|l|l|l|}
\hline \multirow{2}{*}{$\operatorname{Re} / M$} & \multicolumn{4}{|l|}{ Lower plate $(\eta=0)$} & \multicolumn{4}{l|}{ Upper plate $(\eta=1)$} \\
\cline { 2 - 9 } & 1.0 & 2.0 & 3.0 & 4.0 & 1.0 & 2.0 & 3.0 & 4.0 \\
\hline 0.5 & 0.1856 & 0.1434 & 0.1179 & 0.1004 & 0.1897 & 0.1465 & 0.1204 & 0.1025 \\
\hline 1.0 & 0.1828 & 0.1414 & 0.1164 & 0.0992 & 0.1910 & 0.1476 & 0.1214 & 0.1034 \\
\hline 1.5 & 0.1796 & 0.1393 & 0.1147 & 0.0979 & 0.1917 & 0.1485 & 0.1222 & 0.1042 \\
\hline 2.0 & 0.1759 & 0.1369 & 0.1130 & 0.0965 & 0.1920 & 0.1491 & 0.1229 & 0.1048 \\
\hline
\end{tabular}

Table. 3 Variation of skin-friction with upper and lower wall slip parameters

\begin{tabular}{|l|l|l|l|l|l|l|l|l|}
\hline \multirow{2}{*}{$\beta_{1} / \beta_{2}$} & \multicolumn{4}{l}{ Lower plate $(\eta=0)$} & \multicolumn{4}{l|}{ Upper plate $(\eta=1)$} \\
\cline { 2 - 9 } & 0.5 & 1.0 & 1.5 & 2.0 & 0.5 & 1.0 & 1.5 & 2.0 \\
\hline 0.5 & 0.1985 & 0.1941 & 0.1913 & 0.1893 & 0.2074 & 0.2066 & 0.2060 & 0.2056 \\
\hline 1.0 & 0.1961 & 0.1903 & 0.1863 & 0.1834 & 0.2013 & 0.1989 & 0.1972 & 0.1959 \\
\hline 1.5 & 0.1946 & 0.1878 & 0.1828 & 0.1791 & 0.1974 & 0.1937 & 0.1910 & 0.1890 \\
\hline 2.0 & 0.1936 & 0.1859 & 0.1803 & 0.1759 & 0.1947 & 0.1900 & 0.1864 & 0.1838 \\
\hline
\end{tabular}

Table. 4 Variation of rate of heat transfer with Casson parameter and permeability parameter

\begin{tabular}{|l|l|l|l|l|l|l|l|l|}
\hline \multirow{2}{*}{$\beta / K$} & \multicolumn{4}{|l|}{ Lower plate $(\eta=0)$} & \multicolumn{4}{l|}{ Upper plate $(\eta=1)$} \\
\cline { 2 - 9 } & 1.0 & 2.0 & 3.0 & 4.0 & 1.0 & 2.0 & 3.0 & 4.0 \\
\hline 0.25 & -1.6887 & -1.5307 & -1.4789 & - & 0.2051 & 0.4037 & 0.4787 & 0.5184 \\
& & & & 1.4535 & & & & \\
\hline 0.50 & -2.2652 & -1.9592 & -1.8544 & - & - & -0.0782 & 0.0234 & 0.0758 \\
\hline
\end{tabular}




\begin{tabular}{|l|l|l|l|l|l|l|l|l|}
\hline & & & & 1.8013 & 0.3638 & & & \\
\hline 0.75 & -2.7756 & -2.3553 & -2.2118 & - & - & -0.4684 & -0.3446 & -0.2818 \\
\hline 1.00 & -3.2119 & -2.6962 & -2.5216 & - & 2.1389 & 0.8287 & & \\
& & & & 2.4332 & 1.2137 & -0.7871 & -0.6439 & -0.5720 \\
\hline
\end{tabular}

Table. 5 Variation of rate of heat transfer with Reynolds number and Hartmann number

\begin{tabular}{|l|l|l|l|l|l|l|l|l|}
\hline \multirow{2}{*}{$\operatorname{Re} M$} & \multicolumn{6}{|l|}{ Lower plate $(\eta=0)$} & \multicolumn{4}{l|}{ Upper plate $(\eta=1)$} \\
\cline { 2 - 9 } & 1.0 & 2.0 & 3.0 & 4.0 & 1.0 & 2.0 & 3.0 & 4.0 \\
\hline 0.5 & -1.3410 & -1.4379 & -1.5498 & -1.6664 & 0.6695 & 0.4561 & 0.2989 & 0.1654 \\
\hline 1.0 & -1.3753 & -1.4802 & -1.5973 & -1.7182 & 0.5931 & 0.3962 & 0.2442 & 0.1121 \\
\hline 1.5 & -1.4135 & -1.5259 & -1.6481 & -1.7732 & 0.5143 & 0.3339 & 0.1870 & 0.0562 \\
\hline 2.0 & -1.4558 & -1.5752 & -1.7024 & -1.8317 & 0.4333 & 0.2690 & 0.1271 & - \\
& & & & & & & & 0.0025 \\
\hline
\end{tabular}

Table. 6 Variation of rate of heat transfer with upper and lower wall slip parameters

\begin{tabular}{|l|l|l|l|l|l|l|l|l|}
\hline \multirow{2}{*}{$\beta_{1} / \beta_{2}$} & \multicolumn{4}{|l|}{ Lower plate $(\eta=0)$} & \multicolumn{4}{l|}{ Upper plate $(\eta=1)$} \\
\cline { 2 - 9 } & 0.5 & 1.0 & 1.5 & 2.0 & 0.5 & 1.0 & 1.5 & 2.0 \\
\hline 0.5 & -1.3869 & -1.3871 & -1.3873 & -1.3879 & 0.6448 & 0.6704 & 0.6878 & 0.7004 \\
\hline 1.0 & -1.3824 & -1.3805 & -1.3798 & -1.3797 & 0.5978 & 0.6173 & 0.6316 & 0.6424 \\
\hline 1.5 & -1.3799 & -1.3768 & -1.3753 & -1.3746 & 0.5684 & 0.5823 & 0.5931 & 0.6016 \\
\hline 2.0 & -1.3784 & -1.3744 & -1.3723 & -1.3711 & 0.5483 & 0.5574 & 0.5650 & 0.5714 \\
\hline
\end{tabular}

Table. 7 Variation of rate of heat transfer with Peclet number and Eckert number

\begin{tabular}{|l|l|l|l|l|l|l|l|l|}
\hline \multirow{2}{*}{$P e / E c$} & \multicolumn{4}{|l|}{ Lower plate $(\eta=0)$} & \multicolumn{4}{l|}{ Upper plate $(\eta=1)$} \\
\cline { 2 - 9 } & 0.5 & 1.0 & 1.5 & 2.0 & 0.5 & 1.0 & 1.5 & 2.0 \\
\hline 0.2 & -2.0643 & -2.0568 & -2.0493 & -2.0418 & 0.8778 & 0.9771 & 1.0763 & 1.1755 \\
\hline 0.4 & -1.8979 & -1.8809 & -1.8638 & -1.8468 & 0.7674 & 0.8779 & 0.9884 & 1.0988 \\
\hline 0.6 & -1.7323 & -1.7069 & -1.6815 & -1.6561 & 0.6513 & 0.7771 & 0.9028 & 1.0285 \\
\hline 0.8 & -1.5707 & -1.5377 & -1.5047 & -1.4717 & 0.5339 & 0.6800 & 0.8260 & 0.9721 \\
\hline
\end{tabular}

Table. 8 Variation of rate of heat transfer with Prandtl number and heat generation parameter

\begin{tabular}{|l|l|l|l|l|l|l|l|l|}
\hline \multirow{2}{*}{$P r / H$} & \multicolumn{4}{|l|}{ Lower plate $(\eta=0)$} & \multicolumn{4}{l|}{ Upper plate $(\eta=1)$} \\
\cline { 2 - 8 } & 0.02 & 0.04 & 0.06 & 0.08 & 0.02 & 0.04 & 0.06 & 0.08 \\
\hline 0.71 & -1.0452 & -1.1080 & -1.1820 & - & - & -2.5343 & -2.6460 & -2.7906 \\
& & & & 1.2712 & 2.4469 & & & \\
\hline 1.50 & -1.7637 & -1.8815 & -2.0229 & - & - & -4.2208 & -4.4753 & -4.7994 \\
& & & & 2.1961 & 4.0182 & & & \\
\hline 2.50 & -2.6731 & -2.8607 & -3.0874 & - & - & -6.3557 & -6.7908 & -7.3421 \\
\hline 3.50 & -3.5826 & -3.8398 & -4.1519 & - & - & -8.4906 & -9.1063 & -9.8848 \\
\hline
\end{tabular}

Table. 9 Variation of rate of heat transfer with Biot numbers for upper wall and lower wall

\begin{tabular}{|c|c|c|c|c|c|c|c|c|}
\hline \multirow{2}{*}{$B_{1} / B_{2}$} & \multicolumn{4}{|c|}{ Lower plate $(\eta=0)$} & \multicolumn{4}{|c|}{ Upper plate $(\eta=1)$} \\
\hline & 3.0 & 4.0 & 5.0 & 6.0 & 3.0 & 4.0 & 5.0 & 6.0 \\
\hline
\end{tabular}




\begin{tabular}{|c|c|c|c|c|c|c|c|c|}
\hline 0.2 & -1.4152 & -1.3214 & -1.2709 & $-\overline{1.2393}$ & $\begin{array}{l}- \\
0.1240\end{array}$ & -0.1609 & -0.1808 & -0.1932 \\
\hline 0.4 & -1.3695 & -1.2962 & -1.2559 & $-\overline{1.2304}$ & $\begin{array}{l}- \\
0.0642\end{array}$ & -0.1248 & -0.1582 & -0.1794 \\
\hline 0.6 & -1.3365 & -1.2776 & -1.2447 & $-\overline{1.2237}$ & $\begin{array}{l}- \\
0.0210\end{array}$ & -0.0982 & -0.1414 & -0.1689 \\
\hline 0.8 & -1.3116 & -1.2633 & -1.2360 & $-\overline{1.2184}$ & 0.0116 & -0.0777 & -0.1283 & -0.1608 \\
\hline
\end{tabular}

\section{Acknowledgements}

The authors extend their sincere gratitude to editor and reviewers of the journal, for their constructive suggestions and comments for renovation of this article.

\section{Appendix}

$$
\begin{aligned}
& a_{1}=1+\frac{1}{\beta}, a_{2}=M+\frac{1}{K}, m_{1}=\frac{R e-\sqrt{\operatorname{Re}^{2}+4 a_{1} a_{2}}}{2 a_{1}}, m_{2}=\frac{R e+\sqrt{R e^{2}+4 a_{1} a_{2}}}{2 a_{1}}, \\
& a_{3}=\left(1+\beta_{1} m_{1}\right) \exp \left(m_{1}\right), a_{4}=\left(1+\beta_{1} m_{2}\right) \exp \left(m_{2}\right), a_{5}=1-\beta_{2} m_{1}, a_{6}=1-\beta_{2} m_{2}, a_{7}=\frac{P}{a_{2}}, \\
& a_{8}=\frac{\left(a_{4}-a_{6}\right) a_{7}}{a_{3} a_{6}-a_{4} a_{5}}, a_{9}=\frac{\left(a_{3}-a_{5}\right) a_{7}}{a_{3} a_{6}-a_{4} a_{5}}, m_{3}=\frac{P e-\sqrt{P e^{2}-4 H}}{2}, m_{4}=\frac{P e+\sqrt{P e^{2}-4 H}}{2}, \\
& a_{10}=\left(a_{1} m_{1}^{2}+a_{2}\right) a_{8}^{2}, a_{11}=\left(a_{1} m_{2}^{2}+a_{2}\right) a_{9}^{2}, a_{12}=2 a_{8} a_{9}\left(a_{1} m_{1} m_{2}+a_{2}\right), a_{13}=2 a_{2} a_{7} a_{9}, \\
& a_{14}=2 a_{2} a_{7} a_{8}, a_{15}=\frac{a_{10} E c P r}{4 m_{1}^{2}-2 P e m_{1}+H}, a_{16}=\frac{a_{11} E c P r}{4 m_{2}^{2}-2 P e m_{2}+H}, \\
& a_{17}=\frac{a_{12} E c P r}{\left(m_{1}+m_{2}\right)^{2}-\operatorname{Pe}\left(m_{1}+m_{2}\right)+H}, \quad a_{18}=\frac{a_{13} E c P r}{m_{2}^{2}-P e m_{2}+H}, a_{19}=\frac{a_{14} E c P r}{m_{1}^{2}-P e m_{1}+H}, \\
& a_{20}=m_{3}-B_{2}, a_{21}=m_{4}-B_{2}, a_{22}=\left(2 m_{1}-B_{2}\right) a_{15}+\left(2 m_{2}-B_{2}\right) a_{16} \text {, } \\
& a_{23}=\left(m_{1}+m_{2}-B_{2}\right) a_{17}+\left(m_{2}-B_{2}\right) a_{18}, a_{24}=\left(m_{1}-B_{2}\right) a_{19}-B_{2}, a_{25}=a_{22}+a_{23}+a_{24} \text {, } \\
& a_{26}=\left(m_{3}+B_{1}\right) \exp \left(m_{3}\right), a_{27}=\left(m_{4}+B_{1}\right) \exp \left(m_{4}\right) \text {, } \\
& a_{28}=\left(2 m_{1}+B_{1}\right) a_{15} \exp \left(2 m_{1}\right)+\left(2 m_{2}+B_{1}\right) a_{16} \exp \left(2 m_{2}\right), a_{29}=\left(m_{1}+m_{2}+B_{1}\right) a_{17} \exp \left(m_{1}+m_{2}\right), \\
& a_{30}=\left(m_{1}+B_{1}\right) \exp \left(m_{1}\right)+\left(m_{2}+B_{1}\right) \exp \left(m_{2}\right), a_{31}=a_{28}+a_{29}+a_{30}, a_{32}=\frac{a_{25} a_{27}-a_{21} a_{31}}{a_{20} a_{27}-a_{21} a_{26}}, \\
& a_{33}=\frac{a_{20} a_{31}-a_{25} a_{26}}{a_{20} a_{27}-a_{21} a_{26}} .
\end{aligned}
$$

\section{References}

[1] A. Bejan, A study of entropy generation in fundamental convective heat transfer, Journal of Heat Transfer, 101 (1979) 718-725.

[2] A. Bejan, Second law analysis in heat transfer, Energy, 5 (1980) 720-732.

[3] H. Abbassi, M. Magherbi, A. Ben Brahim, Entropy generation in Poiseuille-Benard channel flow, Int. J. Therm. Sci, 42 (2003) 1081-1088. 
[4] O. D. Makinde, E. Osalusi, Second law analysis of laminar flow in a channel filled with saturated porous media, Entropy, 7 (2005) 148-160.

[5] S. Mahmud, R. A. Fraser, Flow, thermal, and entropy generation characteristic inside a porous channel with viscous dissipation, International Journal of Thermal Sciences, 44 (2005) 21-32.

[6] S. Taghizadeh, A. Asaditaheri, Heat transfer and entropy generation of laminar mixed convection in an inclined lid driven enclosure with a circular porous cylinder, International Journal of Thermal Sciences, 134 (2018) 242-257.

[7] M. I. Afridi, M. Qasim, Entropy generation and heat transfer in boundary layer flow over a thin needle moving in a parallel stream in the presence of nonlinear Rosseland radiation, International Journal of Thermal Sciences, 123 (2018) 117-128.

[8] D. Cimpean, I. Pop, A study of entropy generation minimization in an inclined channel, WSEAS Transactions on Heat and Mass Transfer, 6 (2011) 31-40.

[9] A. S. Eegunjobi, O. D. Makinde, Combined effect of buoyancy force and Navier slip on entropy generation in a vertical porous channel, Entropy, 14 (2012) 1028-1044.

[10] T. Chinyoka, O. D. Makinde, A. S. Eegunjobi, Entropy analysis of unsteady magnetic flow through a porous pipe with buoyancy effects, Journal of Porous Media, 16 (2013) 823-36.

[11] S. Das, R. N. Jana, Entropy generation due to MHD flow in a porous channel with Navier slip, Ain Shams Engineering Journal, 5 (2014) 575-584.

[12] G. Hunt, N. Karimi, M. Torabi, Analytical investigation of heat transfer and classical entropy generation in microreactors-the influences of exothermicity and asymmetry, Applied Thermal Engineering, 119 (2017) 403-424.

[13] S. Das, R. N. Jana, A. J. Chamka, Entropy generation in an unsteady MHD channel flow with Navier slip and asymmetric convective cooling, Int. J. Industrial Mathematics, 9 (2017) 149-160.

[14] R. Haq, S. Nadeem, Z. H. Khan, T. G. Okedyao, Convective heat transfer and MHD effects on Casson nano-fluid over a shrinking sheet, Central European Journal of Physics, 12 (2014) 862-871.

[15] T. Poornima, P. Sreenivasulu, N. B. Reddy, Slip flow of Casson rheological fluid under variable thermal conductivity with radiation effects, Heat Trans. Asian Res, 44 (2015) 718-737.

[16] M. H. Aboalbashari, N. Freidoonimehr, F. Nazar, M. M. Rashidi, Analytical modeling of entropy generation for Casson nano-fluid flow induced by a stretching surface, Advanced Powder Technology, 26 (2015) 542-552.

[17] O. D. Makinde, A. S. Eegunjobi, Entropy analysis of thermally radiating magnetohydrodynamic slip flow of Casson fluid in a microchannel filled with saturated porous media, Journal of Porous Media, 19 (2016) 799-810.

[18] M. Gnaneswara Reddy, P. Vijaya Kumari, P. Padma, Effect of thermal radiation on MHD casson nanofluid over a cylinder, Journal of Nanofluids, 7 (2018) 428-438.

[19] N. S. Shashikumar, B. C. Prasannakumara, M. Archana, B. J. Geerisha, Thermodynamics analysis of a Casson nanofluid flow through a porous microchannel in the presence of hydrodynamic slip: a model of solar radiation, Journal of Nanofluids, 8 (2019) 63-72. 
[20] B. Gireesha, C. Srinivasa, N. Shashikumar, M. Macha, J. Singh, B. Mahanthesh, Entropy generation and heat transport analysis of Casson fluid flow with viscous and Joule heating in an inclined porous microchannel, Journal of Process Mechanical Engineering, 233 (2019) 1173-1184.

[21] Ch. Kalyan Kumar, S. Srinivas, Influence of joule heating and thermal radiation on unsteady hydromagnetic flow of chemically reacting casson fluid over an inclined porous stretching sheet, Special Topics \& Reviews in Porous Media: An International Journal, 10 (2019) 385-400.

[22] M. Venkateswarlu, M. Prameela, O. D. Makinde, Influence of heat generation and viscous dissipation on hydromagnetic fully developed natural convection flow in a vertical micro-channel, Journal of Nanofluids, 8 (2019) 1506-1516.

[23] M. Venkateswarlu, P. Bhaskar, D. Venkata Lakshmi, Soret and Dufour effects on radiative hydromagnetic flow of a chemically reacting fluid over an exponentially accelerated inclined porous plate in presence of heat absorption and viscous dissipation, J. Korean Soc. Ind. Appl. Math, 23 (2019) 157-178.

[24] M. Venkateswarlu, O. D. Makinde, P. Rami Reddy, Influence of Hall current and thermal diffusion on radiative hydromagnetic flow of a rotating fluid in presence of heat absorption, Journal of Nanofluids, 8 (2019) 756-766.

[25] M. Venkateswarlu, O. D. Makinde, D. V. Lakshmi, Influence of thermal radiation and heat generation on steady hydromagnetic flow in a vertical micro-porous-channel in presence of suction/injection, Journal of Nanofluids, 8 (2019) 1010-1019.

[26] P. Bhaskar, M. Venkateswarlu, Hall effects on hydromagnetic natural convection flow in a vertical micro-porous-channel, J. Korean Soc. Ind. Appl. Math, 24 (2020) 103-119.

[27] M. Venkateswarlu, D. Venkata Lakshmi, O. D. Makinde, Thermodynamic analysis of Hall current and Soret number on hydromagnetic couette flow in a rotating system with a convective boundary condition, Heat Transfer Research, 51 (2020) 83-101.

[28] G. Manjunatha, C. Rajashekhar, K. V. Prasad, H. Vaidya, B. Saraswati, Peristaltic flow of a Jeffery fluid over a porous conduit in the presence of variable liquid properties and convective boundary conditions, International Journal of Thermofluid Science and Technology, 6 (2019) Paper No. 19060201.

[29] B. Shankar Goud, B. Suresh Babu , M. N Raja Shekar, G.Srinivas, Mass transfer effects on MHD flow through porous medium past an exponentially accelerated inclined plate with variable temperature and thermal radiation, International Journal of Thermofluid Science and Technology, 6 (2019) Paper No. 19060402.

[30] G. Manjunatha, C. Rajashekhar, H. Vidya, K. V. Prasad, B. Saraswati, B. Divya, Heat transfer analysis on peristaltic transport of a Jeffery fluid in an inclined elastic tube with porous walls, International Journal of Thermofluid Science and Technology, 7 (2020) Paper No. 20070101.

[31] M. K. Nayak, A. K. Abdul Hakeem, B. Ganga, Influence of non-uniform heat source/sink and variable viscosity on mixed convection flow of third grade nanofluid over an inclined stretched riga plate, International Journal of Thermofluid Science and Technology, 6 (2019) Paper No. 19060401. 
[32] G. Ibanez, Entropy generation in MHD porous channel with hydrodynamic slip and convective boundary conditions, International Journal of Heat and Mass Transfer, 80 (2015) 274-280. 